\title{
Análise da imagem e esquema corporal em indivíduos com diagnóstico de síndrome pós-poliomielite
}

\author{
Analysis of image and body schema in individuals diagnosed with post-polio syndrome \\ Cintia de Cássia Ferreira ${ }^{1}$, Francis Meire Favero ${ }^{2}$, Sissy Veloso Fontes ${ }^{3}$,
Abraháo Augusto Juviniano Quadros ${ }^{4}$, Sônia Soares Rodrigues Pereira ${ }^{5}$,
Acary Souza Bulle Oliveira ${ }^{6}$, Tânia Fazzini Scheuer
}

\section{RESUMO}

A Síndrome Pós-Poliomielite (SPP) é uma desordem neurológica que se manifesta em indivíduos que tiveram poliomielite anterior aguda, após quinze anos ou mais, caracterizada pela presença de um novo quadro sintomatológico. Dentre os sinais incapacitantes da SPP, destacam-se a diminuição da capacidade funcional com redução no desempenho das AVD's. Este estudo teve como objetivo analisar o Esquema Corporal (EC) em indivíduos com diagnóstico de SPP por meio de um método específico: Procedimento de Marcação do Esquema Corporal ou Image Marking Procedure (IMP). Método. trata-se de um estudo descritivo e transversal com abordagem quantitativa. A amostra foi composta por dez (10) indivíduos com diagnóstico de SPP cadastrados e assistidos no ambulatório de SPP do Setor de Doenças Neuromusculares - UNIFESP/EPM. Resultados. Observou-se que $30 \%$ dos indivíduos apresentaram acometimentos em MMII, 30\% em MID, $10 \%$ em MIE, $10 \%$ em HCE, $10 \%$ em HCD e $10 \%$ em MMII e MSE, percepção menos centralizada do corpo, assimetria corporal, dimensóes corporais mais largas em segmentos corporais menos acometidos pela SPP, percepçáo das alturas das estruturas anatômicas não estavam próxima à altura real. Conclusáo. Os indivíduos desse estudo apresentaram uma percepção alterada em relação as suas dimensôes corporais característica de distúrbio da IEC. Essas alterações estão diretamente relacionadas à sua motricidade.

Unitermos. Doenças Neuromusculares, Distorção da percepção, Imagem Corporal.

Citaçáo. Ferreira CC, Favero FM, Fontes SV, Quadros AAJ, Pereira SSR, Oliveira ASB, Scheuer TF. Análise da imagem e esquema corporal em indivíduos com diagnóstico de síndrome pós-poliomielite.

Trabalho realizado no setor de neuromuscular da disciplina de neurologia clínica - departamento de neurologia e neurocirurgia da Universidade Federal de Sáo Paulo/Unifesp, Sáo Paulo-SP, Brasil.

1.Fisioterapeuta, Especialização pela Unifesp, Sáo Paulo-SP, Brasil.

2.Fisioterapeuta, doutoranda pela Unifesp, São Paulo-SP, Brasil.

3.Professora doutora em neurociência e fisioterapeuta da disciplina de neurologia clinica da Unifesp, São Paulo-SP, Brasil.

4.Mestre em neurociências pela Unifesp e doutorando da disciplina de neurologia da Unifesp, São Paulo-SP, Brasil.

5.Mestre em comunicação pela universidade Mackenzie, especialista em Psicopedagogia clinica e Psicomotricidade, São Paulo-SP, Brasil.

6.Professor doutor em neurologia e neurociências responsável pelo setor de neuromuscular da Unifesp, São Paulo-SP, Brasil.

7.Fisiterapeuta, Especialização pela Unifesp, São Paulo-SP, Brasil.

\begin{abstract}
The post-poliomyelitis syndrome is a neurological disease that manifests in individuals had poliomyelitis, after fifteen years or more, characterized by the presence of new symptoms. Among the incapacities of the PPS, the low function with low performance of the daily activities is detached. This study had as objective to analyze physical projection (PP) in individuals with the diagnosis of PPS by a specific method: Image Marking Procedure (IMP). Method. It is a descriptive and transversal study with quantitative boarding. The sample was composed by ten (10) people with diagnosis of SPP that were registered in the clinic of SPP in the Illnesses Neuromuscular Section- UNIFESP/ EPM. Results. Observed that $30.0 \%$ individuals had presented attack MMII being $30.0 \%$ of MID. The individuals had a low centered perception of their body, body asymmetry, larger body dimensions in body segments less attacked by the PPS, height perceptions of the anatomical structures were not close to the real height. Conclusion. individuals of this study had a modified perception related to its physical dimensions that characterizes the image and physical projection. These alterations are related directly to its movement.
\end{abstract}

Keywords. Neuromuscular Diseases, Perceptual Distortion, Body Image.

Citation. Ferreira CC, Favero FM, Fontes SV, Quadros AAJ, Pereira SSR, Oliveira ASB, Scheuer TF. Analysis of image and body schema in individuals diagnosed with post-polio syndrome.
Endereço para correspondência: Cíntia de Cássia Ferreira Av. Satélite, 486 CEP 08330-480, Santa Bárbara-SP, Brasil E-mail: ccffisioterapia@gmail.com 


\section{INTRODUÇÃO}

A Síndrome Pós-Poliomielite (SPP) é uma desordem neurológica que se manifesta em indivíduos que tiveram poliomielite anterior aguda, após quinze anos ou mais, caracterizada pela presença de um novo quadro sintomatológico. Normalmente, os primeiros sintomas são fadiga, cansaço muscular súbito ou sensação generalizada de exaustão; além de presença de dor muscular e articular com fraqueza muscular progressiva dos músculos que foram ou não originalmente afetados ${ }^{1-5}$. Os indivíduos podem ainda apresentar dispnéia, disfagia, distúrbios no sono, intolerância ao frio, depressão e ansiedade, o que pode interferir diretamente em sua qualidade de vida $(\mathrm{QV})^{1,4,6}$.

A SPP é classificada como neuronopatia motora em virtude do quadro clínico e histológico estar intimamente relacionado com a disfunção dos neurônios motores inferiores $^{2,5}$. O quadro clínico resulta na diminuição da capacidade funcional com redução no desempenho das atividades diárias, particularmente tarefas relacionadas com a mobilidade e surgimento de novas incapacidades ${ }^{1-5}$.

Os novos sinais da doença aparecem preferencialmente nos membros previamente mais fracos e nos indivíduos com maior fraqueza residual. A instalaçáo da síndrome tem íntima relação com a idade de apresentação da doença aguda. Quanto maior a idade, maior a possibilidade de desenvolver a SPP. A piora do quadro clínico é variável de acordo com cada indivíduo. Na maioria dos casos a progressão é lenta, com períodos de estabilização seguida de agravo do quadro clínico. Todas as alteraçóes motoras e funcionais podem interferir na percepção do próprio corpo e comprometer a manutenção da Imagem e Esquema Corporal (IEC) ${ }^{7}$.

A Imagem Corporal (IC) está relacionada com a consciência que o indivíduo tem do seu próprio corpo em termos de julgamento. Como esse indivíduo vê, pensa, sente e age em relação a ele em consideração às normas sociais, valores e atitudes ${ }^{8}$. Possui base afetiva, e é construída e alterada gradativa e permanentemente ao longo da vida' . O termo Esquema Corporal (EC), em contraste, refere-se à representação abstrata em tempo real na linha do próprio corpo no espaço que é derivado do input sensorial. Um sistema neuronal que recebe constantemente informaçóes multissensoriais que incluem a exteriocep- ção (sensibilidade superficial: tátil, dolorosa e térmica), os sistemas proprioceptivos, vestibulares, somatossensoriais e visuais acerca da cinestesia e da posição do corpo no espaço, de maneira a interagir com o sistema motor ${ }^{10}$.

Os indivíduos com SPP apresentam sequelas motoras que podem ou não interferir na manutenção da sua IEC, visto que a análise da IEC pode identificar alteraçóes que influenciam na motricidade voluntária e na sua $\mathrm{QV}$, que de acordo com a World Health Organization (WHO), pode ser definida como "a percepção do indivíduo de sua posição na vida, no contexto da cultura e sistema de valores nos quais ele vive e em relaçáo aos seus objetivos, expectativas, padróes e preocupaçôes" ${ }^{\text {7,9,11,12. }}$.

A alteração em uma parte do corpo, seja por uma doença ou comprometimento físico, resultará em modificações na IEC não apenas referente a essa parte, mas sim ao corpo todo, além de estabelecer novas relaçóes consigo mesmo e com os outros ${ }^{13}$.

Os distúrbios do EC encontrados na SPP levam o indivíduo a apresentar uma percepção alterada em relação as suas dimensóes corporais que podem ser avaliadas por meio do Procedimento de Marcação do Esquema Corporal - IMP, proposto por Askevold que direcionou suas pesquisas para a percepçáo de pontos anatômicos específicos utilizando uma referência autógena a partir da recordação do EC no espelho que será utilizado nesse trabalho ${ }^{7,14}$. Diante do exposto acima, os objetivos gerais deste trabalho foram analisar a IEC em indivíduos com diagnóstico de SPP por meio de um método específico: Procedimento de Marcação do Esquema Corporal ou Image Marking Procedure (IMP) proposto por Askevold para identificação de possíveis alterações da $\operatorname{IEC}^{14}$.

\section{MÉTODO}

Foi realizado estudo de caso, com amostra nãoprobabilística aleatória simples, composta por dez indivíduos com diagnóstico de SPP, que estavam sendo assistidos no Ambulatório de SPP do Setor de Doenças Neuromusculares - UNIFESP. A seleção dos indivíduos foi realizada pela coleta de dados retrospectiva por meio dos prontuários médicos, protocolos de avaliação do ambulatório de SPP e posteriormente, realizado convite aos indivíduos por contato telefônico e pessoalmente durante o atendimento ambulatorial. 
Os critérios de inclusão no estudo foram indivíduos com diagnóstico de SPP, possibilidade motora em MMSS( Amplitude de movimento ativa em flexão, adução e abdução de ombro; flexão e extensão de cotovelo; movimento de pinça fina em indicadores e polegares, para segurar a caneta), ambos os sexos, usuários ou não de dispositivos auxiliares, ser cadastrado a assistido no Ambulatório de SPP, independente de grupo étnico ou classe social. Foram excluídos indivíduos que apresentaram doenças associadas que também causam comprometimento do neurônio motor, alterações visuais e/ou entendimento, cadeirante e indivíduos que não apresentassem interesse em participar como voluntário da pesquisa proposta.

Os voluntários preencheram o Termo de Consentimento Livre e Esclarecido. O projeto foi aprovado pelo Comitê de Ética da UNIFESP no 2099/08.

\section{Procedimento}

A avaliação da IEC foi realizada por meio do IMP baseando-se no protocolo de Askevold ${ }^{15}$.

Os materiais utilizados para as avaliaçóes foram folhas de papel Kraft (1,50m x 1,0 m), canetas hidrográficas de cor vermelha, preta, azul e verde, canetas esferográficas de cor azul, duas réguas - de $50 \mathrm{~cm}(1)$ e de $60 \mathrm{~cm}(1)$, esquadro, trena, fita crepe, venda para os olhos e etiqueta Ink Jet / Laser para a identificação dos indivíduos.

A aplicação do IMP pelo examinador foi realizada em três coletas, individualmente, em uma única sessão, com duração de 20 minutos, de acordo com a descrição dos procedimentos abaixo.

Os indivíduos foram posicionados em bipedestaçẫo diante de uma folha de papel Kraft, fixada à parede de forma que suas mãos atingissem o papel. Foram orientados a realizar o teste de olhos vendados com a seguinte instrução verbal: "imagine que esta folha de papel é um espelho, e você está se vendo nele. Eu vou tocar em alguns pontos do seu corpo e você vai marcar no papel onde você está vendo(imaginando) cada ponto" (Imagem percebida). Quando o indivíduo não entendia a instrução esta era novamente repetida até que a mesma estivesse clara ${ }^{14-16}$. Em seguida, o indivíduo foi posicionado próximo ao papel Kraft, para a marcação da posição real dos mesmos pontos anatômicos pelo pesquisador.

A sequência de pontos anatômicos tocados pelo pesquisador foi a seguinte: extremidade superior da cabeça, juntas acrômio claviculares direita e esquerda, crista ilíaca direita e esquerda, trocanteres femurais.

As medidas das distâncias dos pontos marcados pelo indivíduo e pelo pesquisador foram realizadas tanto no plano horizontal, que representa a largura corporal $^{6,12,17}$, como no plano vertical, que representa a altura entre o real e o percebido em termos de lateralidade, para verificar as assimetrias ${ }^{18}$.

\section{Análise Estatística}

A análise dos dados foi feita quantitativamente com o uso do software estatístico: SPSS (Statistical Package for Social Science) V11.5, Minitab 14 e Excel XP e o nível de significância adotado foi de 5\% de erro. Os dados foram analisados por meio dos testes de Friedman e Wilcoxon.

Utilizou-se testes e técnicas estatísticas não paramétricas, porque as condiçóes (suposiçóes) para a utilização de técnicas e testes paramétricos, como a normalidade (teste de Anderson-Darling, gráfico de distribuição de normalidade, sigla $\mathrm{AD}$ ) e homocedasticidade (homogeneidade das variâncias, teste de Levene), não foram encontradas (principalmente a normalidade) neste conjunto de dados.

Comparou-se as coletas 1, 2 e 3 para cada situação (Tabelas 1 e 2). Nestas análises, foi utilizado o teste de Friedman, uma vez que os dados são pareados.

Calculou-se a diferença de Real para Percebida em altura e largura, a qual foi ponderada pelo Real. Desta forma, obteve-se o percentual da diferença de Real para Percebida. Com base nestes valores, realizou-se uma análise descritiva completa para altura e largura.

\section{RESULTADOS}

Da população pesquisada, seis indivíduos eram do sexo masculino ( $60 \%$ de $\mathrm{N}=10$ ) e quatro do sexo feminino (40\% de $\mathrm{N}=10$ ). A amostra utilizada neste trabalho não apresenta diferença significativa de sexo de acordo com a literatura ${ }^{19}$. A média de idade encontrada foi 41,5 anos, tendo como idade mínima 32 anos e máxima de 53 anos. A mediana de idade é 40 anos. Isso confirma os achados de estudos brasileiros, onde a média de idade encontrada em indivíduos que desenvolveram a SPP foi de 36 e 43,3 anos. 
Tabela 1

Cálculo (média, mediana, desvio padrão, quartis, intervalo de confiança e valor de p) das medidas em cm das larguras reais e percebidas das marcaçôes especificas por estruturas anatômicas de toda a amostra $(N=30)$

\begin{tabular}{ccc|c|c|c|c}
\hline \multirow{2}{*}{ Largura } & \multicolumn{2}{c|}{ Ombros } & \multicolumn{2}{c|}{ Cintura } & \multicolumn{3}{c}{ Trocânter } \\
\cline { 2 - 7 } & Real & Percebida & Real & Percebida & Real & Percebida \\
\hline Média & 31,2 & 41,9 & 30,0 & 41,0 & 34,5 & 42,5 \\
Mediana & 32,0 & 44,0 & 30,1 & 40,5 & 34,0 & 42,9 \\
Desvio Padrão & 3,5 & 9,0 & 2,7 & 6,5 & 3,4 & 10,2 \\
Q1 & 29,4 & 38,7 & 28,7 & 36,7 & 32,2 & 36,3 \\
Q3 & 33,6 & 47,4 & 31,1 & 44,5 & 36,6 & 49,7 \\
N & 30 & 30 & 30 & 30 & 30 & 30 \\
IC & 1,26 & 3,20 & 0,96 & 2,32 & 1,22 & 3,65 \\
p-valor & \multicolumn{2}{c}{$<0,001^{*}$} \\
\hline
\end{tabular}

Tabela 2

Cálculo (média, mediana, desvio padrão, quartis, intervalo de confiança e valor de p) das medidas em cm das alturas reais e percebidas em relação ao solo das marcaçôes específicas por estruturas anatômicas de toda a amostra $(N=30)$

\begin{tabular}{|c|c|c|c|c|c|c|c|c|c|c|}
\hline Altura & & & Média & Mediana & $\begin{array}{l}\text { Desvio } \\
\text { Padráo }\end{array}$ & Q1 & Q3 & $\mathbf{N}$ & IC & p-valor \\
\hline \multirow{2}{*}{ Cabeça } & & Real & 159,4 & 161,7 & 8,7 & 153,1 & 165,3 & 30 & 3,11 & \multirow{2}{*}{$0,002^{*}$} \\
\hline & & Percebida & 153,5 & 153,5 & 11,5 & 146,1 & 162,2 & 30 & 4,10 & \\
\hline \multirow{4}{*}{ Ombros } & \multirow{2}{*}{ Dir } & Real & 130,9 & 132,3 & 7,9 & 124,7 & 136,7 & 30 & 2,84 & \multirow{2}{*}{0,318} \\
\hline & & Percebida & 132,4 & 128,7 & 9,6 & 126,4 & 138,6 & 30 & 3,43 & \\
\hline & \multirow{2}{*}{ Esq } & Real & 131,2 & 132,0 & 8,8 & 125,2 & 137,1 & 30 & 3,14 & \multirow{2}{*}{0,812} \\
\hline & & Percebida & 131,1 & 131,1 & 8,9 & 122,9 & 137,5 & 30 & 3,18 & \\
\hline \multirow{4}{*}{ Cintura } & \multirow{2}{*}{ Dir } & Real & 99,1 & 99,4 & 7,3 & 93,5 & 105,7 & 30 & 2,63 & \multirow{2}{*}{$<0,001^{*}$} \\
\hline & & Percebida & 109,6 & 109,6 & 12,1 & 99,4 & 117,6 & 30 & 4,34 & \\
\hline & \multirow{2}{*}{ Esq } & Real & 99,6 & 101,3 & 8,2 & 92,8 & 106,0 & 30 & 2,93 & \multirow{2}{*}{$<0,001^{*}$} \\
\hline & & Percebida & 107,3 & 107,3 & 22,5 & 96,8 & 120,0 & 30 & 8,04 & \\
\hline \multirow{4}{*}{ Trocânter } & \multirow{2}{*}{ Dir } & Real & 75,3 & 75,5 & 10,1 & 71,4 & 79,5 & 30 & 3,60 & \multirow{2}{*}{$<0,001^{*}$} \\
\hline & & Percebida & 92,5 & 86,6 & 20,1 & 80,3 & 100,9 & 30 & 7,21 & \\
\hline & \multirow{2}{*}{ Esq } & Real & 76,2 & 75,1 & 8,5 & 71,3 & 82,2 & 30 & 3,03 & \multirow{2}{*}{$<0,001^{*}$} \\
\hline & & Percebida & 92,5 & 88,9 & 22,7 & 78,6 & 102,6 & 30 & 8,13 & \\
\hline
\end{tabular}

Em termos de dominância manual, há $70 \%$ de destro e 30\% de canhoto. Quanto ao uso de dispositivos auxiliares, seis indivíduos não faziam uso $(60 \%$ de $\mathrm{N}=10)$ e quatro faziam uso ( $40 \%$ de $\mathrm{N}=10)$. Dentre os indivíduos que faziam uso de dispositivos auxiliares, dois utilizavam bengala em MSD ( $20 \%$ de $\mathrm{N}=10)$, um indivíduo utilizava muleta canadense $(10 \%$ de $\mathrm{N}=10)$ e um indivíduo muleta axilar em MMSS (10\% de N=10). Em relação às sequelas motoras, observa-se, que há um maior acometimento em MMII (30,0\% de $\mathrm{N}=10)$, e MID $(30,0 \%$ de $\mathrm{N}=10)$ no grupo estudado (Tabela 3).
Os resultados obtidos apontam para alteração dentre a população estudada quanto à altura real apenas em trocânter direito. Os resultados obtidos na população estudada quanto á altura, apresentam hiperesquematia, principalmente em trocânter direito.

Com relação à altura da cabeça e larguras corporais, foi calculado o IPC, que corresponde ao tamanho percebido dividido pelo tamanho real multiplicado por 100. Em relação à largura dos ombros, cintura e trocânteres, o grupo apresentou uma hiperesquematia, no entanto, perceberam os segmentos de ombro e cintura mais hiperesquemáticos. 
A análise da altura da cabeça e ombros mostrou que o grupo percebeu a altura próxima a $100 \%$. Em relação à altura da cintura e trocânteres direito e esquerdo, o grupo apresentou uma hiperesquematia, porém em trocânter direito, o grupo percebeu o segmento mais hiperesquemático (Tabela 3).

Tabela 3

Distribuição de sequelas motoras encontradas nos indivíduos $(N=10)$

\begin{tabular}{lcc}
\hline \multicolumn{1}{c}{ SEQUELAS } & QTDE & $\%$ \\
\hline Membros Inferiores (MMII) & 03 & 30,0 \\
Membro Inferior Direito (MID) & 03 & 30,0 \\
\hline Membro Inferior Esquerdo (MIE) & 01 & 10,0 \\
\hline Hemicorpo Direito (HCD) & 01 & 10,0 \\
\hline Hemicorpo Esquerdo (HCE) & 01 & 10,0 \\
\hline $\begin{array}{l}\text { Membros Inferiores (MMII) e } \\
\text { Membro Superior Esquerdo (MSE) }\end{array}$ & 01 & 10,0 \\
\hline
\end{tabular}

$\mathrm{Na}$ análise descritiva dos desenhos do IMP, observamos que os indivíduos com sequela em $H D$, tenderam a deslocar o corpo para o lado contralateral como mostra a Figura 1a (traço em vermelho são as dimensóes percebidas e em preto as reais).

Os que apresentaram maior acometimento de MID tenderam a deslocar a cabeça para o mesmo lado da sequela e apresentam também elevação em trocânter direito (Figura 1b). Apresentaram uma percepção menos centralizada do corpo e uma assimetria corporal como mostra a Figura 2. Quando comparado as larguras dos segmentos corporais, os indivíduos perceberam suas dimensôes corporais para ombro e cintura mais largas em relação à trocânteres, como mostra a figura 2. Em relaçáo à percepção das alturas das estruturas anatômicas cabeça, ombro, cintura e trocânteres, observou-se que estes segmentos não estão próximos à altura real mostrando uma assimetria (Figura 2). Assim como os indivíduos com acometimento em MMII, perceberam os trocânteres mais altos comparado com a altura real (Figura 3).

Os resultados da avaliação da IEC por meio do teste do IMP da largura dos segmentos corporais mostraram uma hiperesquematia. Em ombro e cintura, os indivíduos se perceberam maior quando comparados com o real e em relação aos trocanteres (Figura 2). Já em MMII (trocanteres) esta percepção apresentou um valor mais próximo à $100 \%$ quando comparado com ombro e cintura (Tabela 2). Os resultados obtidos nos levam a acreditar que isso se deva pelo maior comprometimento ser em MMII, onde se perceberam menor do que realmente são mesmo apresentando uma hiperesquematia. Acreditamos que isso se deva pelo fato de haver um menor acometimento em MMSS como mostra a Tabela 1.

A amostra apresentou uma percepção distorcida da altura de trocânteres, onde se encontram acima do tamanho real, assim como a altura da cabeça que mostrou uma percepção também abaixo do tamanho real. $\mathrm{O}$ que nos leva a entender que esses indivíduos apresentaram uma diminuição de sua estatura geral. Os resultados obtidos neste estudo mostraram que não houve relação das distorçôes com o uso de dispositivos auxiliares e dominância manual. Quanto a esta dominância manual, acreditamos ser devido à populaçáo estudada apresentar sequela em MMII.

\section{DISCUSSÃO}

Este estudo se mostrou inédito ao propor um trabalho de análise do EC por meio do teste IMP em indivíduos com diagnóstico de SPP abrindo perspectivas para novas condutas de análise e tratamento para esta população.
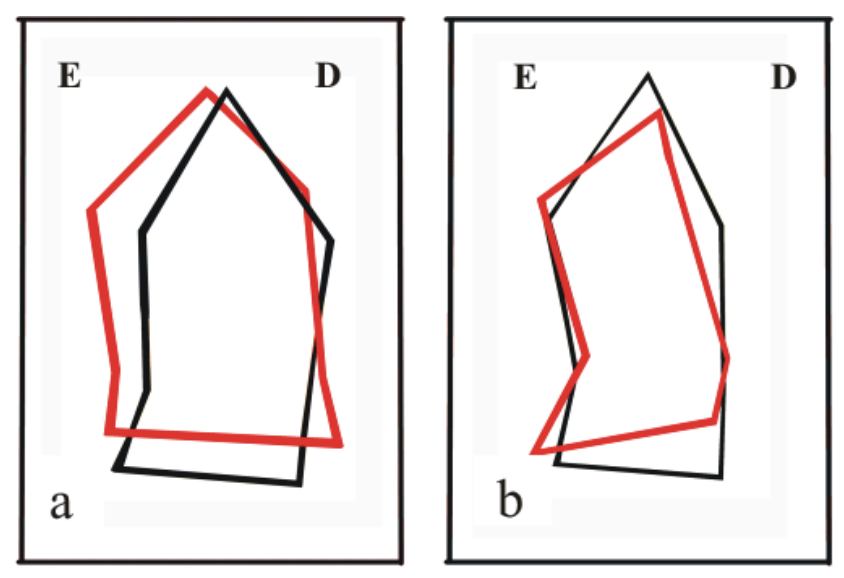

Figura 1. Avaliação da Imagem e Esquema Corporal por meio do teste do Image Marking Procedure.

$1 a$ - acometimento em Hemisfério Direito: mostra o deslocamento do corpo para o lado contralateral; $\mathbf{1 b}$ - acometimento em membro inferior direito: deslocamento da cabeça para o lado ipsilateral à sequela e elevação em trocânter direito.

(traço em vermelho são as dimensôes percebidas e em preto as reais). 


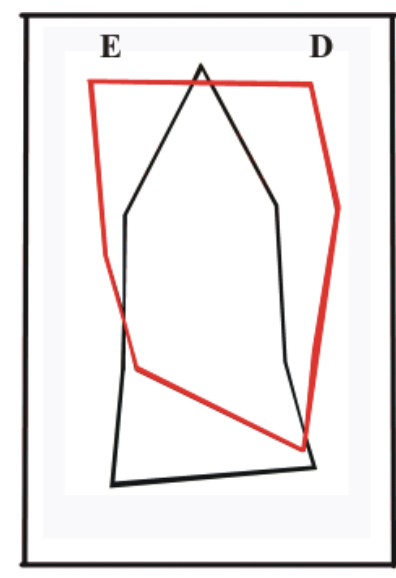

Figura 2. Teste do Image Marking Procedure. Percepção dos segmentos corporais.

Percepção menos centralizada do corpo e uma assimetria corporal das dimensôes corporais para ombro e cintura mais largas em relação à trocânteres e para as alturas das estruturas anatômicas cabeça, ombro, cintura e trocânteres.

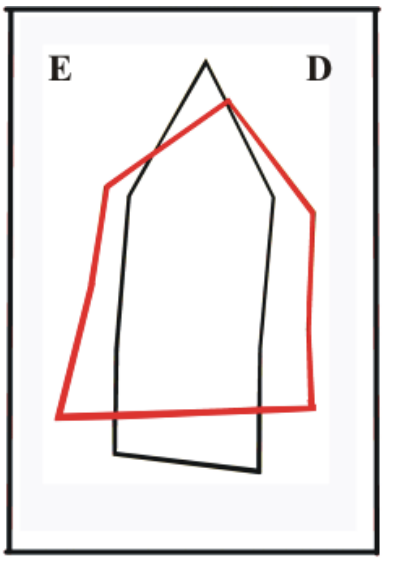

Figura 3. Teste do Image Marking Procedure. Percepçáo dos segmentos corporais.

Acometimento em membros inferiores - percepção dos trocânteres mais altos comparado com a altura real.

Podemos observar que o maior índice de sequelas encontradas localiza-se em MMII e MID com 30,0\%. Estudos mostraram que, o maior acometimento é em MMII (43,7\%), seguido por MID (16,6\%), o que confirma os nossos achados. Este acometimento está de acordo com dados apresentados pelos autores ${ }^{19,20}$. Pesquisadores mostraram que, os MMII também foram os mais acometidos, devido á sobrecarga de trabalho ao longo dos anos e á descarga de peso ${ }^{6}$.

Podemos concluir que a população estudada nesta pesquisa se mostrou homogênea, o que nos permite descrever que não houve diferença significativa para as medidas da altura percebida, largura real e percebida em relação ao solo das marcações específicas por estruturas anatômicas, quando comparado ao cálculo das alturas reais, ou seja, todos os indivíduos se perceberam iguais entre eles de acordo com valores estatísticos.

A percepção do tamanho do corpo está ligada à presença de vagos ou contraditórios EC, tendendo a produzir uma superestimação do tamanho do corpo, mais por deficiência de percepção do que por alteraçóes graves ao nível de $\mathrm{IC}^{10}$. A literatura mostra que a mulher apresenta uma tendência natural em perceber suas dimensóes corporais maiores do que realmente são ${ }^{21}$. O que se observou nesta pesquisa foi uma alteraçáo tanto de altura quanto largura dos segmentos corporais entre a postura real que o corpo adota e a percebida demonstrando que as compensaçóes posturais não são aparentemente percebidas, mas são "incorporadas" à imagem, como se pode observar nesta pesquisa ${ }^{10}$.

A cabeça é um segmento importante para o controle postural, pois possui órgãos sensoriais importantes, ouvido e olhos, para o controle do equilíbrio e a manutenção da postura que tem como função o controle do corpo no espaço e orientação dos segmentos corporais em relação ao meio ambiente e aos próprios segmentos corporais $^{22}$. Em um estudo sobre EC em atletas de alto rendimento e praticantes de atividade física de modalidades diferentes, perceberam a altura da cabeça próxima ao real ${ }^{3}$. Em nossa amostra, apenas dois indivíduos (20\%) realizavam algum tipo de atividade física (caminhada e Yoga). Devido a essa importância funcional essencial que a cabeça tem para o ser humano, acreditamos ser este o motivo do grupo ter percebido com tanta precisáo a altura da cabeça. No entanto, acreditamos também, que quanto mais trabalhamos a questão corporal e exploramos os espaços ao redor do corpo nos permitirá uma melhor percepção corporal. Tais dados concordam com estudos realizados, quando afirma que a IEC envolve a consciência corporal que também estaria relacionada com acontecimentos e vivências anteriores que se tem do próprio corpo $^{23}$.

Estudos mostraram que, seria necessário apresentar sete pontos simétricos, (cabeça, ombros, cinturas e trocanteres) para atingir a totalidade ou simetria absoluta da percepção das dimensôes corporais, o que significaria uma percepção corporal considerada boa e satisfatória e consequente centralização do corpo $^{14}$.

A presença de uma assimetria deve-se a aquisição de mecanismos compensatórios e consequente descentralização corpórea. A literatura mostrou que, a adoção de um mecanismo anormal de manutenção da postura, seria para compensar a deficiência de equilíbrio, o que levaria também á uma assimetria do corpo ${ }^{24,25}$. Essa as- 
simetria e a dificuldade em suportar o peso no lado ou segmento afetado, interferem na capacidade de manter o controle postural, impedindo a orientação e estabilidade para realizar movimentos com o tronco e membros. Cabe ressaltar que a execução das AVD's, tais como vestir-se, alimentar-se, mudar de posição, andar, sentar, alcançar objetos, dependem e envolvem esse controle postural ${ }^{26}$.

A literatura cita a possibilidade de algumas patologias, principalmente as neurológicas e psiquiátricas que podem manifestar distúrbios da IEC, o que foi possível perceber a partir dos resultados obtidos neste estudo ${ }^{27}$.

A importância da percepção corporal está relacionada com a maior atenção sobre as partes integrantes do corpo, e no processo de reabilitação ${ }^{28}$.

De acordo com a literatura pesquisada, a IC deve ser compreendida como um fenômeno singular, estruturado no contexto da experiência existencial e individual do ser humano, em um universo de inter-relaçóes entre imagens corporais. Portanto, entendemos que não podemos falar em distorçâo de IEC, utilizando um único instrumento, pois essa IEC deve ser vista sob múltiplas perspectivas às quais, estão em constantes transformaçóes e integram várias dimensôes, sendo vulneráveis aos processos dinâmicos internos e externos ${ }^{24}$.

Ressaltamos a importância da estimulação da IEC em indivíduos com SPP em próximos estudos, pois a melhora da simetria corporal e melhor controle e centralização do tronco, melhoram o próprio controle postural, os movimentos finos e a marcha ${ }^{22}$. Para os autores, é através das relaçôes de troca com o meio que será possível agregar significados à sua construção pessoal, mantendo assim a integridade de sua IEC que serve como base à execução dos movimentos e conseguirá realizar-se em suas experiências, adquirindo subsídios para o contínuo preenchimento da própria imagem ${ }^{29}$.

Há a necessidade de mais estudos abordando este tipo de trabalho com indivíduos com SPP e com uma população maior, uma vez que a IEC é um sistema neuronal por meio do qual a codificação da ação está centrada na constante renovação de informaçóes multisensoriais acerca do corpo, visto que, para Silva e Martins (2000) o principal objetivo é estimular a percepção, reorientar a atenção visando a independência funcional, no qual o fisioterapeuta deve trabalhar visando á simetria corporal e a facilitação dos movimentos, sendo fundamental um trabalho de estimulação motora e sensorial ${ }^{29}$.

\section{CONCLUSÃO}

Com base nos resultados apresentados, podemos concluir que os indivíduos desse estudo apresentaram uma percepção alterada em relação as suas dimensôes corporais característica de distúrbio da IEC, o que mostrou distorçôes correspondentes a uma hiperesquematia em relação à altura e largura dos segmentos corporais. Para a altura, observou-se que esta hiperesquematia foi maior em trocânter direito, o que confirma nossos achados em relação ao acometimento do segmento corporal e/ou sequela motora. Quanto à largura, essa hiperesquematia encontrou-se com maior ênfase em ombros e crista ilíaca.

Essas alterações estão diretamente relacionadas à sua motricidade e qualidade de vida. Portanto, se faz necessário um maior conhecimento em técnicas de trabalho corporal com uma visão global, que vise atingir não somente o componente estrutural da SPP, mas também o componente psíquico influente na manutenção do sintoma para uma vida útil e adaptada do ponto de vista funcional para esta população.

Para tal, admite-se que o teste do IMP, por não ter nenhum referencial externo que lhe sirva como base, somente os estímulos exteroceptivos e proprioceptivos pode ser utilizado para auxiliar na análise da IEC em indivíduos com SPP. Sendo assim, o uso do método para avaliação da IEC por meio do teste do IMP mostrou-se uma forma terapêutica e diferenciada de avaliar indivíduos com diagnóstico de SPP, podendo enriquecer dados futuros.

\section{REFERÊNCIAS}

1.Oliveira BSA, Quadros JAA, Conde RTM. Documento Técnico da Síndrome Pós - Polio. Associação Brasileira de Síndrome Pós-Polio: ABRASPP. São Paulo, 2004, 31p.

2.Oliveira ASB, Maynard FM. Síndrome Pós-Poliomielite: Aspectos Neurológicos. Rev Neurocienc 2002;10:31- 4.

3.Thorén-Jonsson AL, Grimby G. Distress in everyday life in people with poliomyelitis sequee. J Rehabil Med 2001;33:119-27.

http://dx.doi.org/10.1080/165019701750165952

4.Trojan DA, Cashman NR. Post-poliomyelitis syndrome. Muscle \& Nerve 
2005;31:6-19.

http://dx.doi.org/10.1002/mus.20259

5.Vranjac A. Poliomielite e Síndrome Pós-Poliomielite - Documento Técnico. Divisão de Doenças de Transmissão Hídricas e Alimentares. Centro de Vigilância Epidemiológica. Coordenadoria de Controle de Doenças. Secretaria de Estado da Saúde. São Paulo: Documento elaborado pela DDTHA/CVE com base no parecer SPP, 2006, 23p.

6.Mello A, Marques AP. A imagem corporal representada pelos fibromiálgicos: um estudo preliminar. Rev Fisioter Univ. São Paulo. 1995;2:87-93.

7.Aquino LM. Análise do Esquema e Imagem Corporal em indivíduos com Síndrome Pós-Poliomielite. (Monografia) - São Paulo: Universidade Federal de São Paulo, 2005, 48p.

8.Yuen HK, Hanson C. Body image and exercise in people with and without acquired mobility disability. Disability and Rehabilitation 2002;2(4):6.28996.

9.Peres RS. O Desenho da Figura Humana de Machover aplicado em andarilhos de estrada. Psicologia: Teoria e Prática 2002;4:81-92.

10.Lautenbacher S, Roshcer S, Strian F, Pirke M, Krieg JC. Theoretical and empirical considerations on the relation between body image, body schema and somatosensation. J Psychossom Res 1993;37:447-54.

http://dx.doi.org/10.1016/0022-3999(93)90001-V

11.Fleck MPA. O instrumento de avaliaçáo de qualidade de vida da Organização Mundial da Saúde (WHOQOL-100): características e perspectivas. Ciência da Saúde Coletiva 2000;5:33-8.

12.Machover K. O traçado da Figura Humana: um método para o estudo da personalidade. In: Anderson HH, Anderson GL(Orgs.), Técnicas projetivas do diagnóstico psicológico. São Paulo: Mestre Jou. 1967, p.345-70.

13.Van kolck OL. Técnicas de exame psicológico e suas aplicaçôes no Brasil. Petrópolis, RJ: Vozes, 1981, 432p.

14.Askevold F. Measuring body image. Psycother Psychosom 1975;26:71-7. http://dx.doi.org/10.1159/000286913

15.Fichter MM, Meister I, kcoh HJ. The measurement of body mage disturbances in anorexia nervosa: experimental comparison of different methods. Brit J Psychiat 1986;148:453-61.

http://dx.doi.org/10.1192/bjp.148.4.453
16.Meermann R, Vandereycken W, Napierski C. Methodological problems of body image research in anorexia nervosa patients. Acta Psychiat Belg $1986 ; 86: 42-51$.

17. Maravita A, Iriki A. Tools for the body. Trends in congnitive Sciences 2004;8:79-86.

18.Thurm BE, Gama EF. Efeitos da dor crônica em atletas de alto rendimento em relação ao esquema corporal, agilidade psicomotora e estados de humor. (Tese) - São Paulo: Universidade São Judas Tadeu, São Paulo, 2007,127p.

19.Quadros AAJ, Oliveira ASB, Silva HSA. Síndrome Pós-Poliomielite: Uma Nova Doença Velha. (Tese). São Paulo: Universidade Federal de São Paulo, 2005, 80p.

20.Dalakas MC. The Post-Polio Syndrome as an Evolved Cilinical Entity. Ann NY Acad Sci 1995;753:68-80.

http://dx.doi.org/10.1111/j.1749-6632.1995.tb27532.x

21. Molinari E. Body-size estimation in anorexia nervosa. Percept Motor Skills. 1995;81:23-31.

http://dx.doi.org/10.2466/pms.1995.81.1.23

22.Kandel E, Schwartz J, Jessel T. Princípios da Neurociência. 4.ed. São Paulo: Manole 2003, p.354-454.

23.Schilder P. A imagem do Corpo: as energias construtivas do psique. São Paulo: ed. Martins Fontes Ltda, 1999, 405p.

24.Stokes M. Neurologia para fisioterapeutas. São Paulo: Premier, 2000, 402p. 25.Ribeiro AB, Henriques GR, Corrêa AL, Sanglard RS, Pereira, J.S. A sintomatologia da síndrome de Pusher e o seu impacto no processo de reabilitação: revisão da literatura. Fisioter Bras 2002;3:183-91.

26.Cesário CMM, Penasso P, Oliveira APR. Impacto da disfunção motora na qualidade de vida em pacientes com Acidente Vascular Encefálico. Rev Neurocienc 2006;14:06-9.

27.Lacey J, Birtclmell SA. Body image and it disturbance. Psychosom Res 1986;30:623-31.

http://dx.doi.org/10.1016/0022-3999(86)90096-6

28.Silva CB, Martins JS. Negligência Unilateral: implicaçóes no processo de reabilitação. Rev Fisioter USP. São Paulo, 2000;7:16-24.

29.Monteiro PP. Envelhecer -Histórias. Encontros. Transformações. Belo Horizonte: Autêntica, 2001, 272p. 